\title{
EQUILÍBRIO POSTURAL DINÂMICO É MEDIADO PELA ANTROPOMETRIA E COMPOSIÇÃO CORPORAL EM IDOSAS COM BAIXA DENSIDADE MINERAL ÓSSEA
}

Guilherme Carlos Brech; guibrech@gmail.com; Universidade São Judas Tadeu Jessica Sillas de Freitas; jessicasillas@hotmail.com ; Universidade São Judas Tadeu

Marcia Gouvea; mgouve5@gmail.com; Universidade São Judas Tadeu Adriana Machado-Lima; adrianasaldiba@gmail.com; Universidade São Judas Tadeu Marta Ferreira Bastos; martafbastos@gmail.com; Universidade São Judas Tadeu Liliam Takayama; metosseo@fm.usp.br ; Faculdade de Medicina da Universidade de São Paulo Rosa Maria R. Pereira; rosamariarp@yahoo.com ; Faculdade de Medicina da Universidade de São Paulo Julia Maria D'Andréa Greve; jgreve@usp.br ; Faculdade de Medicina da Universidade de São Paulo Angélica Castilho Alonso; angelicacastilho@msn.com; Universidade São Judas Tadeu

\section{RESUMO}

Objetivo: Investigar a relação da antropometria e composição corporal com equilíbrio postural dinâmico em idosas com baixa Densidade Mineral Óssea (DMO). Métodos: 45 idosas ( $\geq 60$ anos), baixa DMO e diagnóstico nutricional de baixo peso à sobrepeso. Para a avaliação da composição corporal foi utilizado a Densitometria por emissão de raios-x de dupla energia e exame antropométrico para aferir: massa corporal $(\mathrm{kg})$, estatura $(\mathrm{cm})$ e $\mathrm{IMC}(\mathrm{k} / \mathrm{m} 2)$. A avaliação do equilíbrio postural dinâmico foi realizada pelo teste clínico mini Balance Master Evaluation System e o teste computadorizado Balance Master ${ }^{\circledR}$ System pelos testes Sit to Stand e Step Up/Over. Resultados: Houve correlação negativa do miniBESTest $(r=-0,566 ; p \leq 0,001)$ e tempo de subir e descer degrau $(\mathrm{r}=-0,393 ; \mathrm{p} \leq 0,007)$ com a massa gorda, e correlação positiva do miniBESTest $(\mathrm{r}=0,526 ; \mathrm{p} \leq 0,001)$ e tempo de subir e descer um degrau com a massa muscular $(r=0,297 ; p \leq 0,04)$. Quanto às variáveis antropométricas apenas a estatura apresentou correlação positiva $(r=0,296 ; p \leq 0,04)$ com a velocidade no teste de sentar e levantar. Conclusão: A massa magra reduz as oscilações posturais; em contrapartida a massa gorda interferi de forma negativa para o equilíbrio postural dinâmico em mulheres com baixa DMO. A estatura relacionou com o equilíbrio postural dinâmico, quanto mais alta as idosas pior seu equilíbrio.

Palavras-chave: Idosas; Composição corporal; Equilíbrio postural; Densidade mineral óssea.

Agradecimentos: "O presente trabalho foi realizado com apoio da Coordenação de Aperfeiçoamento de Pessoal de Nível Superior - Brasil (CAPES) - Código de Financiamento 001”. 